\title{
VAN DER POL'S EXPRESSIONS FOR THE GAMMA FUNCTION
}

\author{
T. S. NANJUNDIAH
}

1. For the gamma function $\Gamma(z+1)=\Pi(z)$, we have the classical expressions:

$$
\begin{aligned}
& \Pi(z)=e^{-\gamma z} \prod_{k=1}^{\infty} \frac{e^{z / k}}{1+z / k} \\
& \Pi(z)=\prod_{k=1}^{\infty} \frac{(1+1 / k)^{z}}{1+z / k} \\
& \Pi(z)=\lim _{n \rightarrow \infty} n^{z} \prod_{k=1}^{n} \frac{1}{1+z / k}
\end{aligned}
$$

(Weierstrass),

(Gauss).

In the first of these formulas, $\gamma$ denotes the Euler constant. Van der Pol [1] has derived two other new expressions for $\Pi(z)$ : the product

$$
\Pi(z)=\left(\frac{z}{e}\right)^{z} \prod_{k=0}^{\infty} \frac{e_{z+k}}{e_{k}}
$$

and its variant

$$
\Pi(z)=\left(\frac{z}{e}\right)^{z} \lim _{n \rightarrow \infty}(2 \pi n)^{1 / 2} \prod_{k=0}^{n} \frac{e_{z+k}}{e},
$$

with the definitions

$$
e_{z}=\left(1+\frac{1}{z}\right)^{z}, \quad e_{0}=1 .
$$

All the five expressions for $\Pi(z)$ listed above are valid in the whole $z$-plane cut from $-\infty$ to 0 along the negative real axis, the values being principal values. The method adopted in [1] for proving (4) and (5) consists of a transformation of the series for $\log \Pi(z)$ given by (1) as a suitable Stieltjes integral which is then reconverted into two other appropriate series leading respectively to (4) and (5). In the latter case, Stirling's asymptotic representation of $n$ ! is necessarily made use of. Our object in this note is to point out two other alternative methods of proof that seem to be much simpler.

2. If, instead of Weierstrass' product (1), we choose Euler's product (2) for $\Pi(z)$, then (4) can be readily deduced. In fact, this

Received by the editors July 25, 1957. 
is motivated by the argument by which van der Pol obtains a check of Gauss' formula (3) from his own formula (4). Thus raising both sides in the obvious relation

$$
\frac{1+\frac{1}{k}}{1+\frac{z}{k}}=\frac{1+\frac{1}{z+k}}{1+\frac{z}{k+1}}
$$

to the power $z+k$, the result may be written

$$
\frac{\left(1+\frac{1}{k}\right)^{z}}{1+\frac{z}{k}}=\frac{\left(1+\frac{z}{k}\right)^{z+k-1}}{\left(1+\frac{z}{k+1}\right)^{z+k}} \frac{e_{z+k}}{e_{k}}
$$

Here if we take products on both sides for $k \geqq 1$ and make use of (2), we obtain

$$
\mathrm{II}(z)=\left(\frac{z+1}{e}\right)^{z} \prod_{k=1}^{\infty} \frac{e_{z+k}}{e_{k}},
$$

which is the same as (4).

The companion relation (5) is now easily derived by the combined use of (4) and the elementary Stirling formula

$$
n ! \cong\left(\frac{n}{e}\right)^{n}(2 \pi n)^{1 / 2} \quad(n \rightarrow \infty) .
$$

Thus if we define $p(z)$ by setting

$$
\Pi(z)=\left(\frac{z}{e}\right)^{z} p(z)
$$

with the convention

$$
p(0)=1,
$$

we find at once, by making use of the functional equation for $\Pi(z)$,

$$
\frac{h(z)}{p(z+1)}=\frac{e_{z}}{e},
$$

and (6) may be restated as

$$
p(n) \cong(2 \pi n)^{1 / 2} \quad(n \rightarrow \infty) .
$$


Now using (8), we have the relation

$$
1=p(n+1) \prod_{k=0}^{n} \frac{e_{k}}{e}
$$

Here if we let $n \rightarrow \infty$ and utilize (9), we obtain this equivalent of Stirling's formula (6):

$$
1=\lim _{n \rightarrow \infty}(2 \pi n)^{1 / 2} \prod_{k=0}^{n} \frac{e_{k}}{e} .
$$

This together with (4) at once disposes of (5). It should be noted that, if it is only to transform (6) into (10), one could clearly do without $\Pi(z)$ and its functional equation since, for positive integral $z$, (8) is a direct consequence of $(7)$ with $z$ ! in place of $\Pi(z)$.

3. If we use the general Stirling formula

$$
p(z) \cong(2 \pi z)^{1 / 2} \quad(|z| \rightarrow \infty),
$$

holding uniformly in the angular region of the $z$-plane defined by $|\arg z| \leqq \pi-\epsilon$ where $(\pi>) \epsilon>0$ is arbitrary, then a proof of (5) and hence of (4) is immediate with the help of (8). We have, in fact,

$$
p(z)=p(z+n+1) \prod_{k=0}^{n} \frac{e_{z+k}}{e},
$$

giving, on account of (11),

$$
p(z)=\lim _{n \rightarrow \infty}(2 \pi n)^{1 / 2} \prod_{k=0}^{n} \frac{e_{z+k}}{e} .
$$

This proves (5) on substituting into (7). In particular, for $z=0$, (12) coincides with (10) which enables us to transform (12) as

$$
p(z)=\lim _{n \rightarrow \infty} \prod_{k=0}^{n} \frac{e_{z+k}}{e_{k}} .
$$

Inserting (13) into (7), we recover (4).

\section{REFERENCE}

1. B. van der Pol, Note on the gamma function, Canadian J. Math. vol. 6 (1954) pp. 18-22.

Central College, Bangalore, India 